\title{
Right ventricular longitudinal strain is diminished in systemic sclerosis compared with idiopathic pulmonary arterial hypertension
}

\begin{abstract}
To the Editor:
Pulmonary arterial hypertension (PAH) is characterised by severe progressive remodelling of pulmonary arterioles resulting in chronic right ventricular (RV) overload [1]. Regardless of aetiology, RV adaptation to increased pressure load is the main determinant of outcome in PAH [2-4]; however, mortality because of RV failure is significantly higher in systemic sclerosis-related PAH (SSc-PAH) than in idiopathic PAH (IPAH) [5]. Using invasive pressure-volume assessments, we have previously shown that SSc-PAH patients have depressed RV contractility compared with IPAH at similar afterloads [6]. However, the invasive nature of pressure-volume haemodynamics along with the significant expense and technical expertise makes this technique less feasible for routine clinical practice. Although two-dimensional echocardiogram (2DE) is a useful screening tool in PH, RV dysfunction may be often undetected or underestimated, partly because of the complex geometric configuration of the RV chamber and misalignment of the Doppler beam for noninvasive haemodynamic assessment. Speckle-tracking echocardiography (STE), an echo-based imaging modality used in conjunction with $2 \mathrm{DE}$, allows offline calculation of myocardial velocities and deformation parameters in the assessment of regional myocardial contractility [7]. Our group recently demonstrated a heterogeneous pattern of RV longitudinal systolic strain (RVLSS) utilising STE in SSc patients with variable afterloads, which was not appreciable by $2 \mathrm{DE}$ alone [8]. Our findings, however, were shown in a cross-sectional analysis of asymptomatic SSc patients without symptomatic RV failure, right heart catheterisation (RHC)-confirmed PAH or with echocardiographic evidence of RV dilatation. In the current study, we applied this STE-based technique to PAH patients with baseline RV dysfunction to determine whether there were global and regional differences in RVLSS that might distinguish SSc-PAH from IPAH at similar afterload. Our findings have been previously presented in abstract form [9].
\end{abstract}

We prospectively enrolled prevalent and incident IPAH and SSc-PAH patients from September 2007 to November 2015 who underwent $2 \mathrm{DE}$ within $5 \mathrm{~h}$ of RHC. The study protocol was approved by the Johns Hopkins Institutional Review Board, and informed consent was obtained from all patients. PAH was defined by a mean pulmonary artery pressure $(\mathrm{mPAP}) \geqslant 25 \mathrm{mmHg}$ and pulmonary vascular resistance (PVR) $>3 \mathrm{WU}$ (Wood units) with pulmonary artery wedge pressure $\leqslant 15 \mathrm{mmHg}$ at RHC [10]. SSc patients met the American College of Rheumatology/European League Against Rheumatism diagnostic criteria for [11]. Patients with significant chronic obstructive or interstitial lung disease, portal hypertension, severe obstructive sleep apnoea, left-sided heart failure or chronic thromboembolic disease were excluded. Echocardiograms were performed using Phillips ie33 ultrasound (Phillips Healthcare, Andover, MA, USA) according with American Society of Echocardiography guidelines [12]. Two echocardiographers blinded to patient information and clinical variables performed 2DE analysis using FUJIFILM Medical Systems Synapse Cardiovascular Software (V4.0.8.), and commercially available vendor-independent strain software (Epsilon, EchoInsight, Milwaukee, WI, USA) for STE $[7,13]$.

Data are expressed as mean \pm standard error, absolute numbers or percentages. Comparisons between groups were performed with unpaired sample t-test and Wilcoxon-Mann-Whitney test for nonparametric

@ERSpublications

Speckle tracking echo is a useful clinical tool to distinguish differential RV contractility between PAH subtypes http://ow.ly/6Wyr30fVTeu

Cite this article as: Mukherjee M, Mercurio V, Tedford RJ, et al. Right ventricular longitudinal strain is diminished in systemic sclerosis compared with idiopathic pulmonary arterial hypertension. Eur Respir J 2017; 50: 1701436 [https://doi.org/10.1183/13993003.01436-2017]. 
TABLE 1 Demographic information, comorbidities, clinical characteristics, haemodynamics and echocardiographic findings between idiopathic pulmonary arterial hypertension (IPAH) and systemic sclerosis-related pulmonary arterial hypertension (SSc-PAH) patients

All PAH (n=55) IPAH (n=23) SSc-PAH (n=32) $\begin{gathered}\text { p-value (IPAH } \\ \text { versus SSc-PAH) }\end{gathered}$ Normal value

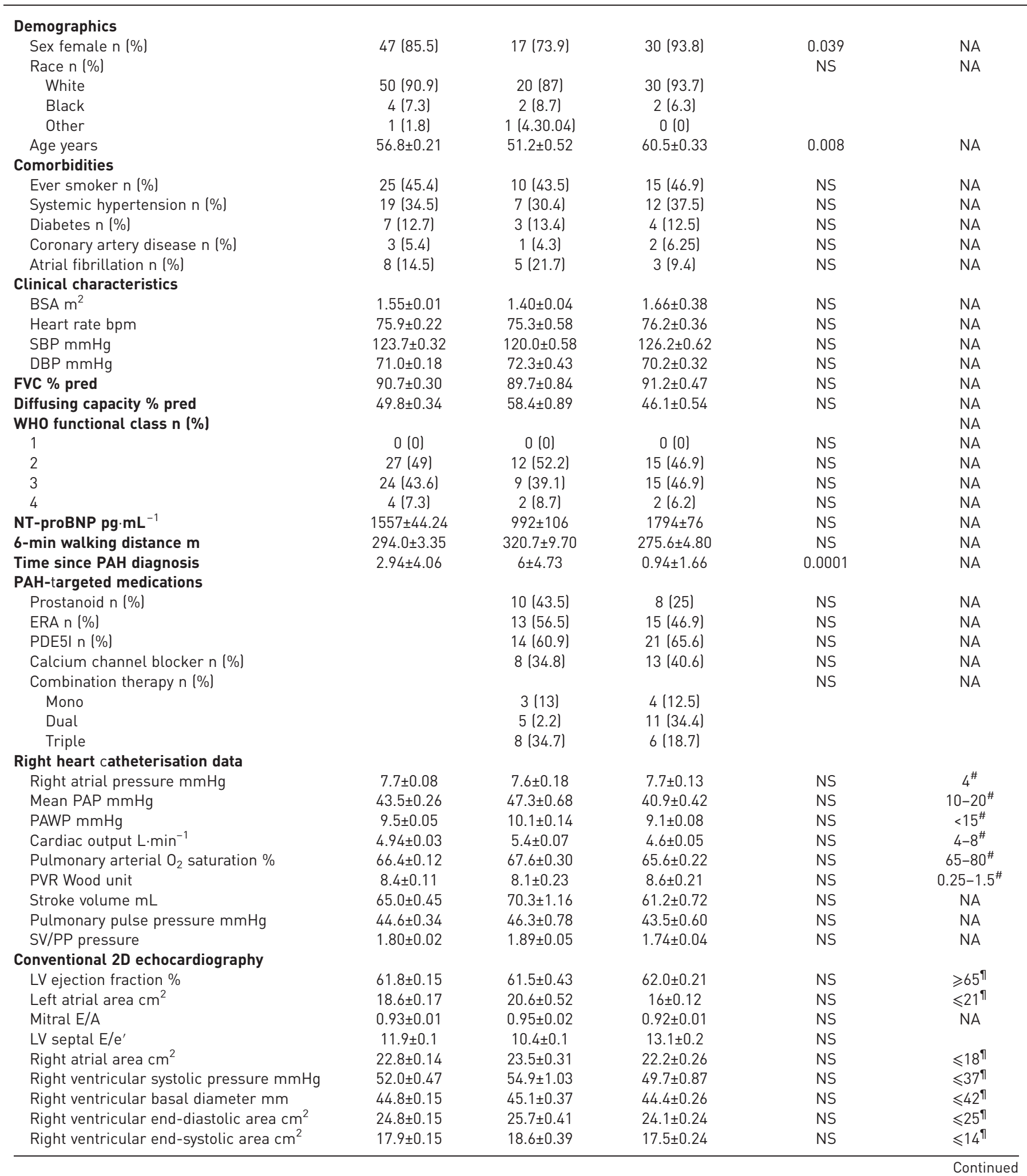


TABLE 1 Continued

\begin{tabular}{|c|c|c|c|c|c|}
\hline & All PAH (n=55) & IPAH (n=23) & SSc-PAH (n=32) & $\begin{array}{c}\mathrm{p} \text {-value (IPAH } \\
\text { versus SSc-PAH) }\end{array}$ & Normal value \\
\hline Fractional area change $\%$ & $17.6 \pm 0.28$ & $17.4 \pm 0.71$ & $17.7 \pm 0.46$ & NS & $\geqslant 35^{\pi}$ \\
\hline Pericardial effusion n (\%) & & & & NS & NA \\
\hline Mild & $14(25.4)$ & $7(30.4)$ & $7(21.9)$ & & \\
\hline Moderate-severe & 4 (7.3) & $0(0)$ & $4(12.5)$ & & \\
\hline Midventricular RVLSS \% & $-13.8 \pm 0.12$ & $-13.8 \pm 0.28$ & $-13.8 \pm 0.22$ & NS & $-27 \pm 5^{+}$ \\
\hline Apical RVLSS \% & $-8.4 \pm 0.10$ & $-9.8 \pm 0.26$ & $-7.4 \pm 0.14$ & NS & $-24 \pm 6^{+}$ \\
\hline Global RVLSS \% & $-13.7 \pm 0.09$ & $-15.6 \pm 0.19$ & $-12.6 \pm 0.16$ & 0.03 & $-26 \pm 4^{+}$ \\
\hline \multicolumn{6}{|c|}{$\begin{array}{l}\text { Data are expressed as mean } \pm \text { standard error. PAH: pulmonary arterial hypertension; NA: not applicable or not available; NS: nonsignificant; } \\
\text { BSA: body surface area; SBP: systolic blood pressure; DBP: diastolic blood pressure; FVC: forced vital capacity; WHO: World Health Organization; } \\
\text { NT-proBNP: N-terminal pro B-type natriuretic peptide; ERA: endothelin receptor antagonist; PDE5I: phosphodiesterase } 5 \text { inhibitor; PAP: } \\
\text { pulmonary artery pressure; PAWP: pulmonary artery wedge pressure; PVR: pulmonary vascular resistance; SV: stroke volume; PP: pulmonary } \\
\text { pulse; LV: left ventricular; TAPSE: tricuspid annular plane systolic excursion; RVLSS: right ventricular longitudinal systolic strain. \#: normal values } \\
\text { taken from [14]; }{ }^{\uparrow}: \text { normal values taken from [12]; }{ }^{+} \text {: normal values taken from [15] (data expressed as mean } \pm \text { standard deviation). }\end{array}$} \\
\hline
\end{tabular}

data and Chi-squared for dichotomous data. Pearson correlation was performed to evaluate for cross-sectional correlation between echocardiographic measurements and haemodynamics. Survival analysis is demonstrated with Kaplan-Meier curves. Statistical analysis was performed using SPSS (Version 22.0, IBM Corp, Armonk, NY, USA).

Our cohort consisted of 55 PAH patients, 23 IPAH and 32 SSc-PAH (31 limited SSc, one diffuse SSc). IPAH patients had longer PAH disease duration in comparison to SSc-PAH. Further clinical and demographics details are described in table 1. Both PAH groups had similar elevation of right atrial pressure, $\mathrm{mPAP}$ and PVR. Stroke volume and pulmonary arterial pulse pressure did not differ between groups. Both PAH groups had normal left ventricular systolic function and, despite the SSc-PAH patients being older, had similar left atrial areas and degrees of diastolic dysfunction. Both groups had similar evidence of right-sided chamber enlargement and diminished 2D derived measures of RV contractility. Global RVLSS (GRVLSS) was markedly diminished in both PAH groups compared with normative values [15], but was significantly worse in SSc-PAH $(p=0.03)$. Both PAH groups demonstrated a heterogeneous pattern of RV contractility with increased basal RVLSS compared with midventricular and apical segments. However, in SSc-PAH there was diminished basal RVLSS compared with IPAH, which was unchanged after adjusting for age, gender, mPAP and PVR. Intra- and inter-reader agreement of RVLSS was excellent. After a mean follow-up period of $88 \pm 1.2$ months, we observed 18 deaths, with a survival rate of $67 \%$ at 5 years. We dichotomised PAH patients according to a GRVLSS less than the average, or more than or equal to the average value of our PAH cohort of $-13.7 \%$. Patients with GRVLSS $<-13.7 \%$ had reduced long-term survival in comparison to patients with GRVLSS $\geqslant-13.7 \%$ (figure 1), and these findings were consistent after adjustment for age and gender. Our study was not powered to demonstrate the predictive value of speckle-derived measures versus conventional measures of RV contractility in PAH; however, it does demonstrate the prognostic value of diminished GRVLSS across PAH subtypes.

To our knowledge, we are the first to reveal significant regional differences in RV function between SSc-PAH and IPAH at similar afterloads. We previously demonstrated a heterogeneous pattern of RVLSS in a large cohort of SSc patients in which there was increased contractility of the basal RV free wall and diminished contractility of the midventricular and apical segments [8]. Here, we similarly demonstrated regional heterogeneity in which midventricular and apical segments had decreased RVLSS compared with the basal RV segment. However, in our current cohort of PAH patients with RV dysfunction, there was diminished GRVLSS in SSc-PAH that was largely driven by decreased basal RVLSS. Importantly, regional abnormalities in contractility detected by RVLSS were not evident by conventional 2DE parameters of RV function such as tricuspid annular plane systolic excursion and fractional area change. We further demonstrated that reduction in GRVLSS of less than $-13.7 \%$ was strongly predictive of long-term survival. However, there were no differences in the predictive value of GRVLSS between IPAH and SSc-PAH potentially signalling a similar mortality outcome once strain derangements reach a certain threshold of abnormality. GRVLSS is not only an important predictor of outcome as we have also demonstrated that RVLSS may improve after initial upfront specific therapy with ambrisentan and tadalafil in SSc-PAH [16]. 


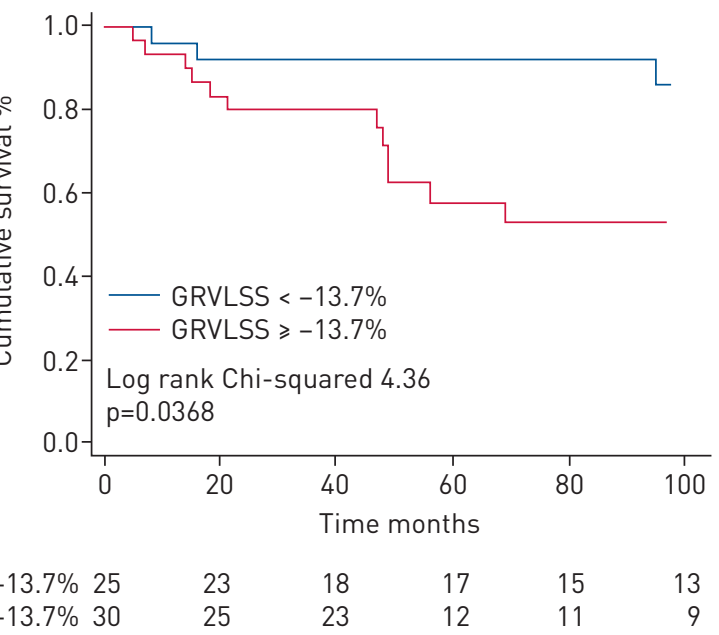

FIGURE 1 Global right ventricular longitudinal systolic strain as a predictor of survival in our cohort of pulmonary arterial hypertension (PAH) patients. Kaplan-Meier curves of survival lall-cause mortality) in PAH patients according to global right ventricular longitudinal systolic strain (GRVLSS) $<-13.7 \%$ or $\geqslant-13.7 \%$.

In summary, we demonstrate significant global and regional differences in RV function between IPAH and SSc-PAH, which were independent of afterload, driven by diminished systolic strain of the basal RV free wall segment in SSc-PAH. Importantly, RV contractile abnormalities as detected by RVLSS were present despite shorter PAH disease duration in SSc-PAH patients. Our findings however suggest differential RV myocardial disease that distinguishes $\mathrm{PAH}$ subtypes that may be detected noninvasively, and may be attributable to myocardial fibrosis and/or microvascular disease unique to SSc. The potential prognostic value of RV strain changes over time in response to therapy will require further assessment through carefully designed longitudinal studies.

Monica Mukherjee ${ }^{1}$, Valentina Mercurio ${ }^{2}$, Ryan J. Tedford ${ }^{1}$, Ami A. Shah ${ }^{3}$, Steven Hsu ${ }^{1}$, Christopher J. Mullin ${ }^{2}$, Takahiro Sato ${ }^{2}$, Rachel Damico ${ }^{2}$, Todd M. Kolb ${ }^{2}$, Stephen C. Mathai ${ }^{2}$ and Paul M. Hassoun ${ }^{2}$

${ }^{1}$ Division of Cardiology, Johns Hopkins University School of Medicine, Baltimore, MD, USA. ${ }^{2}$ Division of Pulmonary and Critical Care Medicine, Baltimore, MD, USA. ${ }^{3}$ Division of Rheumatology, Johns Hopkins University School of Medicine, Baltimore, MD, USA.

Correspondence: Monica Mukherjee, Johns Hopkins University Division of Cardiology, 301 Mason Lord Drive, Suite 2400, Baltimore, MD 21224. E-mail: mmukher2@jhmi.edu

Received: July 102017 | Accepted after revision: Sept 012017

Support statement: Scleroderma Foundation (M. Mukherjee, S.C. Mathai), R01HL114910 and U01HL125175 (P.M. Hassoun), and Italian Society of Cardiology-Merck Sharp \& Dohme Grant (V. Mercurio). Funding information for this article has been deposited with the Crossref Funder Registry.

Conflict of interest: None declared.

\section{References}

1 Hassoun PM. The right ventricle in scleroderma (2013 Grover Conference Series). Pulm Circ 2015; 5:3-14.

2 Campo A, Mathai SC, Le Pavec J, et al. Hemodynamic predictors of survival in scleroderma-related pulmonary arterial hypertension. Am J Respir Crit Care 2010; 182: 252-260.

3 Tyndall AJ, Bannert B, Vonk M, et al. Causes and risk factors for death in systemic sclerosis: a study from the EULAR Scleroderma Trials and Research (EUSTAR) database. Ann Rheum Dis 2010; 69: 1809-1815.

4 Vonk-Noordegraaf A, Haddad F, Chin KM, et al. Right heart adaptation to pulmonary arterial hypertension: physiology and pathobiology. J Am Coll Cardiol 2013; 62: D22-D33.

5 Fisher MR, Mathai SC, Champion HC, et al. Clinical differences between idiopathic and scleroderma-related pulmonary hypertension. Arthritis Rheum 2006; 54: 3043-3050.

6 Tedford RJ, Mudd JO, Girgis RE, et al. Right ventricular dysfunction in systemic sclerosis-associated pulmonary arterial hypertension. Circ Heart Fail 2013; 6: 953-963.

7 Marwick TH, Leano RL, Brown J, et al. Myocardial strain measurement with 2-dimensional speckle-tracking echocardiography: definition of normal range. JACC Cardiovasc Imaging 2009; 2: 80-84.

8 Mukherjee M, Chung SE, Ton VK, et al. Unique abnormalities in right ventricular longitudinal strain in systemic sclerosis patients. Circ Cardiovasc Imaging 2016; 9: e003792.

9 Mukherjee M, Mercurio V, Tedford RJ, et al. Regional heterogeneity of the right ventricle in systemic sclerosis versus idiopathic pulmonary arterial hypertension. Am J Respir Crit Care 2017; 195.

10 Galiè N, Simonneau G. The fifth world symposium on pulmonary hypertension. J Am Coll Cardiol 2013; 62: D1-D3. 
11 van den Hoogen F, Khanna D, Fransen J, et al. 2013 classification criteria for systemic sclerosis: an American College of Rheumatology/European League against Rheumatism collaborative initiative. Arthritis Rheum 2013; 65: $2737-2747$.

12 Lang RM, Badano LP, Mor-Avi V, et al. Recommendations for cardiac chamber quantification by echocardiography in adults: an update from the American Society of Echocardiography and the European Association of Cardiovascular Imaging. Eur Heart J Cardiovasc Imaging 2015; 16: 233-270.

13 Blessberger $\mathrm{H}$, Binder T. Non-invasive imaging: two dimensional speckle tracking echocardiography: basic principles. Heart 2010; 96: 716-722.

14 Bangalore S, Bhatt DL. Images in cardiovascular medicine. Right heart catheterisation, coronary angiography, and percutaneous coronary intervention. Circulation 2011; 124: 428-433.

15 Fine NM, Chen L, Bastiansen PM, et al. Reference values for right ventricular strain in patients without cardiopulmonary disease: a prospective evaluation and meta-analysis. Echocardiography 2015; 32: 787-796.

16 Mercurio V, Mukherjee M, Tedford RJ, et al. Improvement in right ventricular strain with ambrisentan and tadalafil upfront therapy in scleroderma pulmonary arterial hypertension. Am J Respir Crit Care Med 2017; in press [https://doi.org/10.1164/rccm.201704-0789LE]. 